\title{
EVALUACIÓN INTEGRADA A LAS ENFERMEDADES PREVALENTES DE LA INFANCIA
}

\section{INTEGRATED ASSESSMENT OF THE PREVAILING CHILDHOOD DISEASES}

\author{
Roxana Argueta-Álvarez \\ Licenciada en Laboratorio Clínico \\ Investigadora | Universidad de Oriente \\ rargueta@univo.edu.sv
}

\section{Resumen}

El Objetivo de este trabajo consiste en definir las enfermedades más frecuentes en la infancia de los niños y niñas de la Colonia la Presita I del municipio de San Miguel, para ello se empleó la metodología de alcance descriptivo y con enfoque cuantitativo, en la que participaron 32 niños entre las edades de 0-9 años. Este trabajo considera los principios éticos elementales destacando el previo consentimiento informado de los padres de cada uno de los niños, respetando el derecho de confidencialidad. Los resultados principales evidencian que, al realizarles la evaluación psicomotriz a través de la escala simplificada (permite la evaluación del desarrollo de las niñas y niños) el total de ellos y ellas se encuentran realizando adecuadamente las actividades que según su edad es pertinente; tres de estos 32 niños y niñas evidenciaron retardo en su crecimiento, acompañado de un proceso de desnutrición. Entre las patologías más frecuentes en esta población sobrellevadas en los últimos años se pueden mencionar enfermedades gastrointestinales y respiratorias. Brindar al niño y la niña oportunidades para un desarrollo adecuado es el legado más importante que se puede ofrecer a la humanidad. Un desarrollo infantil pleno, principalmente durante los primeros años de vida, sentará las bases para la formación de un sujeto con todo su potencial y con posibilidades de desarrollo.

Palabras clave: Crecimiento, desarrollo psicomotriz, evaluación integral.

\section{Abstract}

The main purpose of this study is to define the most common diseases in children from Colonia la Presita I, in the community of San Miguel. The methodology employed in this study had a descriptive significance and a quantitative approach, where 32 children in between 0-9 years old participated. This study follows the basic ethical principles of informed consent from parents and respects the children's right to confidentiality. Results showed that after psychomotor assessment using a simplified scale (which allows the assessment of child 
development) all of them showed enough ability to carry out the relevant activities that they were supposed to be doing according to their age; three out of the 32 children revealed an alarming growth delay, together with malnutrition. Among the most frequent pathologies in this population during the last years, we could highlight gastrointestinal and respiratory diseases. Giving opportunities to children for an appropriate development is the most important legacy that we can leave for humanity. Healthy development of the infants, especially during the early years of child development, will ensure the formation of potential individuals with development opportunities.

Keywords: Growth, psychomotor development, comprehensive assessment

\section{Introducción}

La situación actual de niñas y niños en su primera infancia impone como desafíos para la familia, el Estado salvadoreño y las instancias corresponsables del cumplimiento de derechos, potenciar el rol protagónico de las familias, cerrar las brechas de acceso y cobertura de servicios $y$ atenciones para la primera infancia, generar ambientes $y$ entornos sanos y seguros, y denunciar y defender los derechos que han sido amenazados o vulnerados (2) . La mortalidad de niñas y niños en edades de 0 a 5 años también se asocia a bajos niveles de salubridad y la falta de acceso a servicios básicos como agua potable y servicios de alcantarillado; el no contar con vivienda en condiciones higiénicas y una adecuada alimentación inciden directamente en enfermedades del sistema digestivo y respiratorio.

La Agenda 2030 para el Desarrollo Sostenible, aprobada en septiembre de 2015 por la Asamblea General de las Naciones Unidas, establece una visión transformadora hacia la sostenibilidad económica, social y ambiental de los 193 Estados Miembros que la suscribieron y es la guía de referencia para el trabajo de la institución en post de esta visión durante los próximos 15 años. En mayo del año 2018, el Instituto Nacional de Salud, por medio del Departamento de Investigaciones en Salud y con el objetivo de "Desarrollar investigaciones científicas e intervenciones en salud que contribuyan en la toma de decisiones"; realizó un proceso de actualización de la Agenda Nacional de Investigación en Salud. Se establecieron, así, 20 líneas de investigación. En ellas, las enfermedades no transmisibles y la nutrición y seguridad alimentaria ocupan el primer lugar y tercero respectivamente. (5) Éstas están relacionadas multifactorialmente a otras condiciones que propician patologías en la niñez, como lo confirma el Consejo de Seguridad Alimentaria y Nutricional (CONASAN) en la 
Política de Seguridad Alimentaria y Nutricional 2018-2028.

El Salvador presenta todavía problemas de desnutrición crónica o retardo en el crecimiento, así como deficiencias de micronutrientes. Además, en las últimas décadas ha sufrido un incremento acelerado de sobrepeso y obesidad, manifestado en los distintos grupos de población a lo largo del ciclo de vida (4), lo cual está generando un aumento de las enfermedades crónicas no transmisibles (hipertensión, enfermedades cardíacas, hiperlipidemias y otras). Existe un problema de anemias por deficiencia de hierro que aún constituye un problema de salud pública especialmente en la población infantil, convirtiéndose en un desafío para el país. (6)

\section{Metodología}

Este estudio fue de alcance descriptivo y con enfoque cuantitativo. Según HernándezSampieri y otros, los estudios descriptivos son aquéllos que buscan especificar las propiedades, características y perfiles de personas, grupos, comunidades, procesos, objetivos - cualquier otro fenómeno que se someta a un análisis. Los estudios cuantitativos, por su parte, son aquéllos que nos ofrecen la posibilidad de generalizar los resultados más ampliamente, nos otorgan control sobre los fenómenos, así como un punto de vista de conteo y las magnitudes de éstos. Asimismo, nos brindan una gran posibilidad de réplica y un enfoque sobre puntos específicos de tales fenómenos, además de que facilitan la comparación entre estudios similares. (I2). Para este estudio se utilizaron técnicas que permitieron la evaluación integral del estado de salud de cada niño y niña entre los cuales se utilizaron exámenes físicos y de evaluación nutricional. Para ello, se llevaron a cabo evaluaciones de peso/talla, evaluaciones de peso/edad, evaluaciones de talla/edad y medio de perímetro craneoencefálico. Se realizaron pruebas químicas especiales como: examen general de orina, examen general de heces y pruebas hematimetrías. Los instrumentos que se utilizaron para este estudio fueron: Tablas percentiles (curvas de crecimiento), Cintas para medir perímetro craneal y Tabla de valores hematimétricos.

\section{Análisis e interpretación de resultados}

A continuación, se presentan los resultados del estudio Evaluación integrada a las enfermedades prevalentes de la infancia, donde se muestran datos generales y clínicos de cada uno de los niños que estuvieron presentes en la investigación. 


\section{a. Datos sociodemográficos:}

Tabla I: Datos sociodemográficos de los participantes

\begin{tabular}{|c|c|c|}
\hline Datos generales & Frecuencia & Porcentaje \\
\hline Edad & 2 & $6 \%$ \\
\hline $0-I$ año & 6 & $19 \%$ \\
\hline I-2 años & 6 & $19 \%$ \\
\hline $3-5$ años & 18 & $56 \%$ \\
\hline $6-9$ años & 32 & $100 \%$ \\
\hline Total & 13 & $41 \%$ \\
\hline Sexo & 19 & $59 \%$ \\
\hline Masculino & 32 & $100 \%$ \\
\hline Femenino & & \\
\hline Total & & \\
\hline
\end{tabular}

Fuente: Elaboración propia

Considerando la distribución de la población en estudio en base a su edad, el $44 \%$ de ellos están en el periodo de la primera infancia y resto en la etapa de la segunda infancia, con una participación casi equitativa entre niños y niñas según su sexo.

\section{b. Evaluación psicomotriz}

Tabla 2: Evaluación psicomotriz

\begin{tabular}{|c|c|c|}
\hline Evaluación psicomotriz & Frecuencia & Porcentaje \\
\hline Adecuado & 8 & $100 \%$ \\
\hline Riesgo & 0 & $0 \%$ \\
\hline Total & 8 & $100 \%$ \\
\hline
\end{tabular}

Fuente: Elaboración propia

Para detectar y atender problemas referentes a los niños durante los primeros dos años de su vida, se utilizaron instrumentos internacionales. Entre ellos, una escala simplificada del desarrollo, que ayudan a la evaluación de parámetros como el desarrollo psicomotriz (la maduración de la estructura nerviosa cerebro, médula, nervios y músculos), que comprende el aprendizaje y habilidades básicas que deben tener los niños durante esta etapa, como moverse, hablar o identificar a sus padres y otras personas. Para esta evaluación se establecieron parámetros desde Adecuado hasta En riesgo, según la cantidad de actividades que el niño realiza. 
Del total de niños que participaron en el estudio el $25 \%$ de ellos oscilan entre estas edades (0-2 años) y al realizarles la evaluación psicomotriz a través de la escala simplificada, el total de ellos se encuentran realizando adecuadamente las actividades que según su edad es pertinente.

\section{c. Evaluación nutricional}

Tabla 3: Peso talla de los niños.

\begin{tabular}{|c|c|c|}
\hline Evaluación nutricional & Frecuencia & Porcentaje \\
\hline Peso & & \\
\hline Normal & 29 & $91 \%$ \\
\hline Desnutrición & 2 & $6 \%$ \\
\hline Desnutrición severa & $\mathrm{I}$ & $3 \%$ \\
\hline Total & & \\
\hline Talla & 32 & $100 \%$ \\
\hline Talla alta & & \\
\hline Normal & 30 & $94 \%$ \\
\hline Retardo en el crecimiento & $\mathrm{I}$ & $3 \%$ \\
\hline Retardo severo en el crecimiento & $\mathrm{I}$ & $3 \%$ \\
\hline & & \\
\hline Total & 32 & $100 \%$ \\
\hline
\end{tabular}

Fuente: Elaboración propia

La evolución del percentil infantil es una de las claves para medir la evolución y desarrollo del niño, y sirve para detectar posibles problemas de crecimiento. Asimismo, se puede saber también si el niño está bajo de peso, lo que puede representar un problema en la etapa del estirón, o padece obesidad infantil, una enfermedad sobre la que alertan agencias sanitarias.

Es importante reflexionar que existen tres de estos 32 niños con una alarmante señal de retardo en el crecimiento, acompañado de un proceso de desnutrición que manifiesta crisis no solo financiera sino alimentaria, con la que se lucha a diario, porque a pesar de que son niños de familias que viven en zona urbana, el déficit alimentario es evidente, no solo por tenerla en la mesa sino saber qué especificadamente se debe comer. 


\section{d. Morbilidad infantil}

Ilustración I: Patologías más frecuentes en la infancia. Fuente: Información recolectada a través del estudio

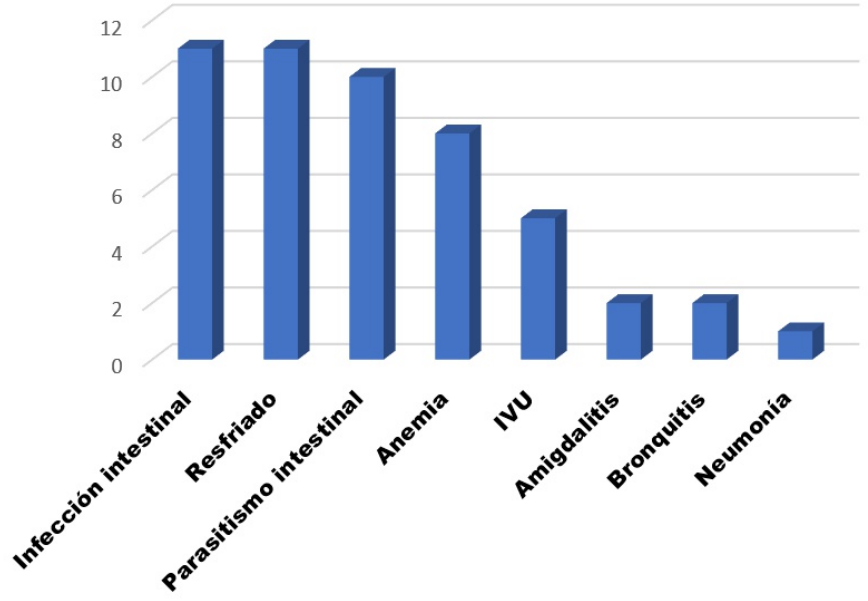

Fuente: Elaboración propia

Se considera morbilidad infantil al número o estadística de niños enfermos o víctimas de una enfermedad en un determinado tiempo. Considerando la valoración integral de los niños como referente de las patologías más frecuentes que han sobrellevado durante los últimos años, es importante mencionar que las enfermedades gastrointestinales y respiratorias son las más prevalentes, la mayoría de ellas leves, muy habituales y típicas de la primera infancia. Es necesario reconocer las patologías respiratorias complicadas, que de alguna manera son frecuentes, como la bronquitis y neumonía.

e. Infecciones relacionadas a las vías urinarias e intestinal

Tabla 4: Resultados general de heces

Resultado

\begin{tabular}{r}
\hline Infección intestinal \\
\hline Infección intestinal \\
Negativo
\end{tabular}

Total
Frecuencia

\begin{tabular}{|c|c|}
\hline 1 & $3 \%$ \\
\hline 5 & $16 \%$ \\
\hline 26 & $81 \%$ \\
\hline 32 & $100 \%$ \\
\hline
\end{tabular}

Fuente: Elaboración propia 
Ilustración 2: Resultados examen general de orina

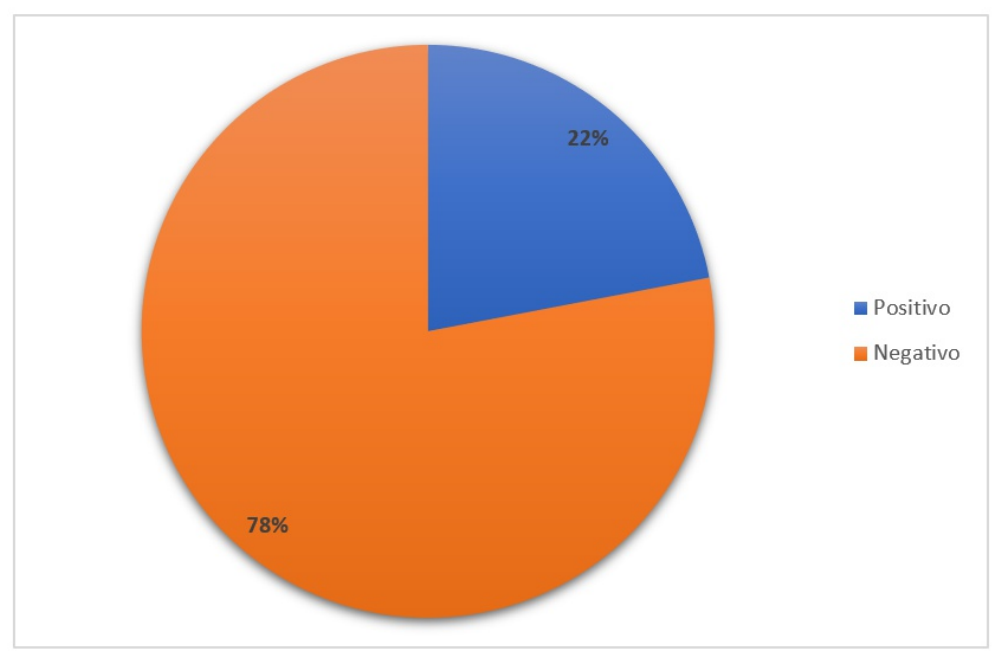

Fuente: Elaboración propia

Las pruebas de examen general de orina y examen general de heces nos sirven para así averiguar o descartar las posibles causas de algunas enfermedades o facilitar el diagnóstico de las mismas a través del examen químico y microbiológico.

Para este estudio los niños, aunque en pocas proporciones, resultaron positivos los exámenes de orina en un $22 \%$, y en $19 \%$ el general de heces, diagnosticando infecciones presentes en los niños tanto de origen bacteriano como parasitario, considerado con un factor fundamental en la calidad de vida de los mismos.

\section{f. Evaluación hematimétrica}

Ilustración 3: Resultados hematimétricos

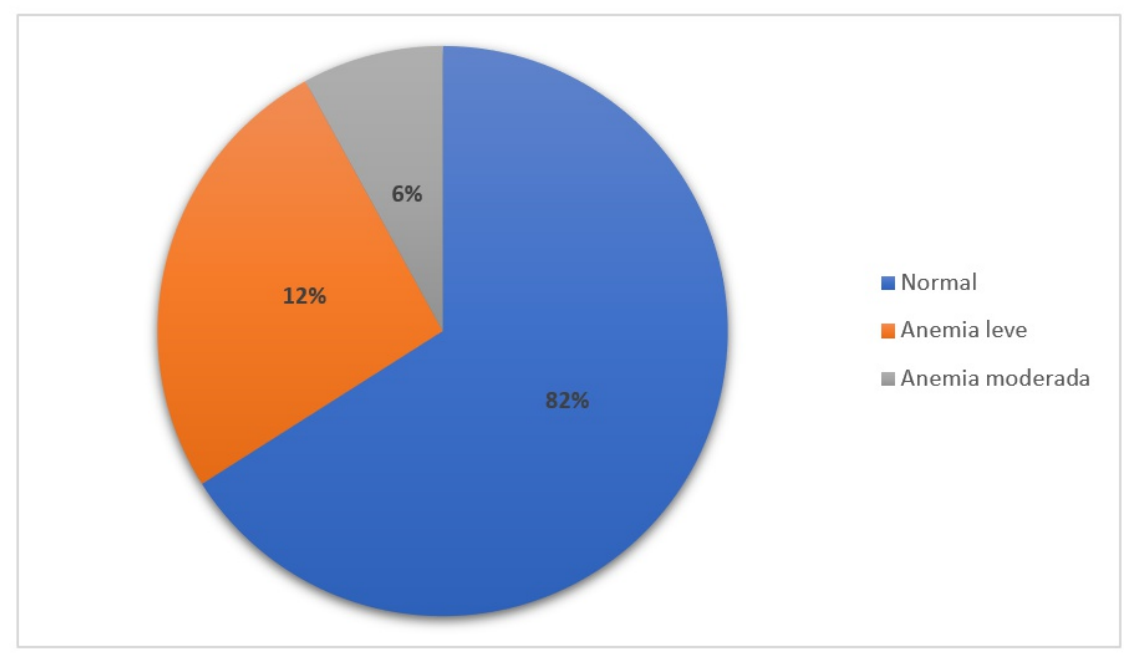

Fuente: Elaboración propia 
El hemograma es una prueba que sirve para orientar hacia el diagnóstico de diversas enfermedades que se han sospechado por la historia clínica y la exploración física.

Considerando los niños que en este estudio resultaron con otros parámetros que se podrían relacionarse con los valores hematimétricos son la talla y peso; aunque en una pequeña proporción resultaron con déficit en estos valores. El resultado del hemograma se relaciona, ya que un $18 \%$ de ellos presentan resultados bajos de hemoglobina, clasificándolos en anemias desde leve a moderada

\section{Discusión}

Todo niño o niña precisa tener condiciones estimulantes para prosperar. El proceso de desarrollo es influenciado no sólo por el estado de salud y nutricional del niño, sino también, por sus propias características biológicas. Asimismo, hoy día se conocen más a fondo las fases específicas del desarrollo intelectual en la primera infancia, que repercuten en los resultados relativos a la salud a lo largo del ciclo vital. Los ambientes enriquecidos y la calidad de la estimulación, la seguridad y el apoyo durante las etapas más sensibles del crecimiento, revisten gran importancia, desde que el niño nace hasta que cumple los ocho años. Los entornos responsables de promover condiciones enriquecedoras para la niñez van desde el universo íntimo de la familia hasta el contexto socioeconómico que vive cada país.

En el año 2007, con el objetivo de conocer la magnitud de la desnutrición proteico-energética en la localidad de Harlem (Pinar del Río), Cuba, e identificar algunos factores de riesgo, se realizó un estudio transversal descriptivo donde se estudiaron los 38 niños que presentaban valores de peso para la talla y peso, para las edades inferiores al tercer percentil. Se encontró un $18,4 \%$ de desnutrición, más frecuente en niños mayores de I año, en los que representó $21,12 \%$.

En este este estudio se puede observar que los niños menores de dos años a los cuales se les evaluó su desarrollo psicomotriz mostraron valores normales. Sin embargo, no todos evidenciaron valores normales al evaluárseles el peso y la talla. El $9 \%$ mostró valores inferiores a la escala y con diagnóstico de desnutrición, y el $6 \%$ evidenció retardo en el crecimiento. Ello que repercute en el estado de salud, que se manifiesta en el índice morbilidad infantil, que considera las enfermedades gastrointestinales y respiratorias, $y$ problemas anémicos.

Un estudio realizado en Colombia en 2014 arrojó resultados similares a este estudio y 
concluyó que el retraso del crecimiento es causa y consecuencia de la pobreza. Los estudios longitudinales que permiten conocer las transiciones entre estados de salud $y$ enfermedad muestran que los efectos acumulados de la desnutrición en la niñez se traducen en la edad adulta en baja productividad, menores ingresos económicos y problemas de salud. Los efectos acumulados de la desnutrición en la niñez también son intergeneracionales: los niños de mujeres que fueron desnutridas durante el inicio de su vida son más pequeños que los hijos de mujeres bien nutridas.

\section{Conclusiones}

De acuerdo al análisis e interpretación de datos obtenidos en la presente investigación se concluye:

"Considerando que la mayoría de los niños se encuentran inscritos en el control de niños sanos en la Unidad de Salud, existen otros factores agregados con la mal nutrición y condiciones de vida, que permiten la prevalencia de patologías como anemia, parasitismo intestinal, infección intestinal, IVU, resfriado, neumonía y amigdalitis, que deterioran su calidad de vida.

"La evolución nutricional es un factor muy importante, considerando que existe en esta población un porcentaje, aunque bajo, de niños con problemas de desnutrición, que puede afectar directamente el desarrollo correcto de los niños.

"Los niños menores de dos años que participaron en este estudio se encuentran en valores normales, al evaluar actividades y habilidades relacionados a la motricidad.

\section{Recomendaciones}

"Para el crecimiento y desarrollo de los niños deben las instituciones de salud brindar atención esencial inmediatamente después del parto, de modo que le permita contribuir a su salud.

"Los padres de familia son responsables de que cada niño, desde recién nacidos, reciba asistencia en salud para recibir una atención adecuada en los centros y establecimientos de salud correspondientes.

"Los establecimientos de salud deben tener en todo momento personal bien formado y motivado para prestar servicios sanitarios a la madre y al niño en todas las etapas de su vida.

"Todas las mujeres y recién nacidos deben tener un historial clínico completo, exacto y estandarizado que permita controlar los procesos de crecimiento y desarrollo de cada uno 
para prevenir problemas que repercutan en la salud.

\section{Bibliografía}

Albaladejo, R., Villanueva, R., Ortega, P., Astasio, P., Calle, M., \& Dominguez, V. (Julio-Agosto de 2004). Revista Española de Salud Pública, Scielo. Obtenido de http://scielo.isciii.es/scielo.php?script=sci_arttext\&pid=SII3557272004000400008

Consejo Nacional de Seguridad Alimentaria y Nutricional. CONASAN. (2018). Política Nacional de Seguridad Aimentaria y Nutricional. 4I.

Fondo de la Naciones Unidas para la Infancia. (3I de Mayo de 20I8). https://www.unicef.org. Obtenido de Estrategia Nacional para el Desarrollo Integral de la Primera Infancia : https:/www.unicef.org/elsalvador/media/ |43 |/file/Estretegia\%20Nacional\%20para\%20el\%20Desarrollo\%20de\%20la\%20primera\%20infancia.pdf

Hernández Sampieri, R., Méndez Valencia , S., Mendoza Torres, C., \& Cuevas Romo, A. (20I7). Fundamentos de Investigación (primera edición ed.). Ciudad de México: Mc.GRAW-HILL/INTERAMERICANA EDITORES, S.A. DE C.V.

Instituto Nacional de Salud, INS. (mayo de 2018). Agenda Nacional de Prioridades de Investigación en Salud 20182014. San Salvador. Obtenido de http://ins.salud.gob.sv/investigacion/

Ministerio de Salud, El Salvador. (marzo de 20I4). "La estrategia AIEPI a disminuido la mortalidad en la niñez". Obtenido de https://w2.salud.gob.sv/novedades/noticias/noticias-ciudadanosas/272-febrero-20|4/2302--|8-0220I4-la-estrategia-aiepi-ha-disminuido-la-mortalidad-en-la-ninez.html

Ministerio de Salud, Instituto Nacional de Salud. (Octubre de 20I7). Manual de Prodedimientos de Investigación en Salud. San Salvador, El Salvador.

Organización Panamericana de la Salud. (20II). http://wwwl.paho.org. Recuperado el 2019 de agosto de 28, de Manual para la vigilancia del desarrollo infantil en el contexto de la AIEPI: http://wwwl.paho.org/hq/dmdocuments/ manual-vigilancia-desarrollo-infantil-aiepi-20I I.pdf

Walter L. Arias Gallegos I, A. M. (diciembre de 2017). Síndrome de burnout en personal de salud de la ciudad de Arequipa (Perú). Scielo. Obtenido de http://scielo.isciii.es/scielo.php?script=sci_arttext\&pid=S0465546X201700040033। 